\title{
A NOTE ON DEDEKIND RINGS
}

\author{
RYÛKI MATSUDA
}

\begin{abstract}
We prove the following theorem: let $R$ be a commutative ring without zero-divisors. If every ideal in $R$ is a product of prime ideals $\neq R, R$ has the identity.
\end{abstract}

To prove the theorem stated in the abstract, let $R$ be a ring satisfying the hypotheses and $K$ its quotient field. We denote $(R: R)_{K}$ by $R^{-1}$. The facts $R^{-1} \ni 1$ and $R^{-1} R=R$ are immediate by the definition. If $a R^{-1}$ coincides with $R$ for all $a \neq 0$ of $R$, we have $R=a^{2} R^{-1}=a R$.

Then we have $a=a b$ for some $b$ of $R$. We see that $b$ is the identity of $R$. We may suppose therefore that $d R^{-1}$ is a proper subset of $R$. Since $d R^{-1}$ is an ideal, there exist prime ideals $\mathfrak{p}_{1}, \mathfrak{p}_{2}, \cdots, \mathfrak{p}_{n}$ such that

$$
d R^{-1}=\mathfrak{p}_{1} \mathfrak{p}_{2} \cdots \mathfrak{p}_{n} \quad(n \geqq 1) .
$$

There exist prime ideals $\mathfrak{g}_{1}, \mathfrak{g}_{2}, \cdots, \mathfrak{g}_{m}$ also such that $\mathfrak{p}_{n} R=\mathfrak{g}_{1} \mathfrak{g}_{2} \cdots \mathfrak{g}_{m}$. Every $\mathfrak{g}_{i}$ contains $\mathfrak{p}_{n}$ and $\mathfrak{p}_{n}$ contains $\mathfrak{g}_{j}$ for some $j$, say 1 . If $\mathfrak{p}_{n} R$ is a proper subset of $\mathfrak{p}_{n}$, we have

$$
\mathfrak{p}_{n} R=\mathfrak{p}_{n} \mathfrak{g}_{2} \cdots \mathfrak{g}_{m} \quad(m \geqq 2) .
$$

Multiplying $d^{-1} R \cdot \mathfrak{p}_{1} \mathfrak{p}_{2} \cdots \mathfrak{p}_{n-1}$ on the both sides, we have $R^{2}=R \mathrm{~g}_{2} \cdots$ $\mathfrak{g}_{m}$. There arises the contradiction of $R \subset \mathfrak{g}_{m}$. Therefore $\mathfrak{p}_{n} R$ coincides with $\mathfrak{p}_{n}$. We have

$$
d R^{-1} R=\mathfrak{p}_{1} \mathfrak{p}_{2} \cdots \mathfrak{p}_{n} R=\mathfrak{p}_{1} \mathfrak{p}_{2} \cdots \mathfrak{p}_{n}=d R^{-1} .
$$

Therefore we see that $R^{-1} R$ is $R^{-1}$, hence we have

$$
R=R^{-1} R=R^{-1} \ni 1 \text {. }
$$

This concludes the proof of our assertion.

Department of Mathematics, Ibaraki University, Mito, Ibaraki, Japan

Received by the editors August 15, 1973.

AMS (MOS) subject classifications (1970). Primary 13F05.

(c) American Mathematical Society 1974 